\title{
Choice of SMAs for damping applications in Civil Engineering: simulations and realistic experiments
}

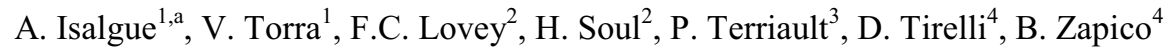 \\ ${ }^{1}$ Dep. Física Aplicada UPC. Campus Nord, B4. Barcelona 08034, Spain \\ ${ }^{2}$ Div. Metales, Centro Atómico Bariloche e Instituto Balseiro, S.C. Bariloche, 8400, Argentina \\ ${ }^{3}$ Dep. de Génie Méchanique, École de Technologie Supérieure, 1100 Notre-Dame Ouest, Montréal Qc, Canada \\ ${ }^{4}$ Lab. ELSA, TP. 480, Joint Research Center EU, Ispra 21020 (VA)-Italia
}

\begin{abstract}
There have been published many papers and several reviews on the application of SMA's as damping devices in civil engineering by using the pseudo-elastic effect, but they are difficult to apply because the behaviour of the alloys is not carefully analyzed. In particular, the summer-winter temperature effects, aging long time at low temperature, self-heating or residual strains requirements for the given applications are rarely well established. In this work, we analyze and compare two cases, the damping of earthquake effects on relatively small structures as family homes, and the damping of stayed cables in large bridges. The requirements for the materials are different, and in each case a different commercial alloy with appropriate conditioning might provide acceptable answers. To confirm the usefulness of the dampers, the hysteretic behaviour of two SMAs (CuAlBe and NiTi) has been analyzed, and later modelled and introduced in finite element codes for the simulation of structures. The behaviour of the structures without and with dampers have been analyzed and compared. Also, some realistic experiments with cable 1 in the ELSA Joint Research Centre of the EU in Ispra have been done with NiTi wire of $2.46 \mathrm{~mm}$ diameter as damper, to compare with simulations.
\end{abstract}

\section{Introduction}

The properties of SMA (or smart materials), are associated to a first order phase transition named martensitic transformation, that occurs between metastable phases from the upper temperature phase (austenite) to the lower temperature phase (martensite). The first order transformation (in coordinates stress, deformation and temperature) has a hysteresis and permits different levels of applications, i.e., actuators and dampers [1]. To work as a damper, the most efficient way would be to absorb a large amount of energy in mechanically transforming the alloy, and then, part of this energy is not returned to the structure, because of the hysteresis, i.e., describing the pseudo-elastic cycle. Two types of applications can be considered in damping of structures in Civil Engineering. The first one is related to the reduction of the damage induced by earthquakes, several papers and reviews have been published on this subject $[2,3]$. The second one is the increase of the lifetime for stayed cables in bridges.

The requirements for damping earthquakes might be stated as, after an unknown waiting time, a damping device must be able to absorb energy from a strong externally induced vibration (the earthquake), reducing considerably the amplitude of the oscillation as the structure suffers as low damage as possible. Then, a large waiting time might be many years, or decades, in which the temperature might change, at least from daily and seasonal (summer-winter) variations. In these conditions, pre-stressing of the SMA, which might be advantageous as any movement is done inside the coexistence of phase's zone, with relevant hysteresis, is not advisable, because of stabilisation of the martensite. If there were a short waiting time before the event, prestressing could be applied, as is done usually in some medical devices or in satellite launching. As an earthquake lasts only around a hundred seconds of large input, and the implied normal frequencies of structures are in the range of one Hertz, the needed fatigue life is less than a thousand cycles, including the major replicas For these conditions, it was found that $\mathrm{CuAlBe}$ was a suitable alloy [4].

a-mail: antonio.isalgued.upc.edu

This is an Open Access article distributed under the terms of the Creative Commons Attribution-Noncommercial License (http://creativecommons.org/licenses/by-nc/3.0/), which permits unrestricted use, distribution, and reproduction in any noncommercial medium, provided the original work is properly cited. 
On the other hand, the damping of the oscillations of stayed cables in bridges, presents a different pattern: The normal frequencies of interest are also from near one Hertz to some tenths of $\mathrm{Hz}$, but a relatively short waiting time is expected (some days or weeks), and a considerable working time is needed, i.e., a storm might last some days. Then, many hundreds of thousands of cycles have to be damped, so the fatigue life must be of near the million cycles, and CuAlBe is not suitable for a so large number of fatigue cycles.

In this work, NiTi is checked for the conditions it has to fulfil for damping the stayed cables of bridges. In particular, the conditions of reliability and fatigue, simulations with finite element (FE) code for stayed cable in the Iroise bridge (Brest to Plougastel, France), and simulations and comparison with experiments on the instrumented cable \#1 at ELSA (Joint Research Centre EU, Ispra, Italy) are done.

\section{Methodology}

The following steps are described in the applied methodology:

-Identification of the problem and its critical points

-Behaviour of NiTi SMA regarding the application

-Simulations:

-Of the SMA characteristics and inclusion in ANSYS code

-Of the instrumented cable \#1 at ELSA and comparison with measurements

-Conclusions

-Of the system (Iroise bridge cable)

\subsection{Identification of the problem and critical points}

Stayed cables in bridges might oscillate due to weather conditions (wind, rain) or to traffic actions. The normal modes start at near one Hertz, and a bad weather period might last several days, so some hundreds of thousands of cycles with considerable amplitude might occur. Then, one of the critical points is the large fatigue life required to have a reliable damper. Other critical point is the changes in ambient temperature, might be slightly more than $20^{\circ} \mathrm{C}$ (from night to day). In any case, direct sun light should be avoided to reduce the temperature span at which the damper is exposed, as the Clausius-Clapeyron coefficient for the used NiTi wires (in our case, $2.45 \mathrm{~mm}$ diameter pseudo-elastic wire from SAES-Getters, and previously from Special Metals) is $6.5 \mathrm{MPa} / \mathrm{K}$ [5], so a change of $20^{\circ} \mathrm{C}$ in ambient temperature mean a change in the stress to transform a sample of $130 \mathrm{MPa}$.

\subsection{Behaviour of NiTi SMA regarding the application}

The general thermo-mechanical behaviour of SMA, specially the pseudo-elasticity, may be applied to damping, as described in [6]. There are some changes in the transformation temperature with low temperature aging. For the pseudo-elastic $\mathrm{NiTi}$, at the temperature of $373 \mathrm{~K}$, a 3 month period would represent a change in transformation temperature of some $6^{\circ} \mathrm{C}$, equivalent to a change in stress to transform of near $40 \mathrm{MPa}$ [7]. This value is not very high, but the actual measurements do not provide a satisfactory boundary for the maximum evolution possible, so care must be taken if the samples would be kept at relatively elevated ambient temperatures for long time.

The acceptable number of cycles before failure increases as the maximum stress decreases, and low stresses (near $200 \mathrm{MPa}$ ) allow reaching more than one million cycles for the $2.46 \mathrm{~mm}$ diameter pseudo-elastic wire [8]. The associated strain is relatively low (less than $1.5 \%$ ), and the hysteresis in one cycle is equivalent to dissipate around $100000 \mathrm{~J} / \mathrm{m}^{3}$.

\subsection{Simulation of the SMA characteristics}

As a first approach to the SMA behaviour for the damping of stayed cables, it is assumed that all actions will occur in a limited period of time. Then, long time effects are included as initial changes on the actual transformation temperature, and given the time scales [7], it is assumed that during the experiments no further significative changes will occur. The self-heating of the material due to the dissipated energy might be also included by performing, as needed, simulations with the estimated extreme temperatures (and then, it is not needed to solve the thermal diffusion along the wire and the heat exchange with the surroundings). Also, for a first approach, we use a bilinear model of the stress-strain behaviour. This has been developed previously in the routine frame of USERMAT in the ANSYS code [4]. A model of some bilinear elements in parallel can represent very accurately the behaviour of the SMA wire (see fig. 9 of [4]), but a first approach can be performed in much less time with the bilinear model. Also, in the OPENSEES (Open System for Earthquake Engineering 
Simulation, see http://opensees.berkeley.edu) code, the bilinear model can be implemented easily by the superposition of some elastic-plastic models as that of STEEL01, by choosing appropriately the parameters to approach the SMA pseudo-elastic cycle, and setting the accumulative damage to zero.

\subsection{Experiments and simulation of the cable \#1 at ELSA}

To verify the behaviour of the simulations, some experiments were done on cable \#1 at ELSA (Joint Research Centre, EU, Ispra, Italy), see fig. 1. The cable has a length of $45 \mathrm{~m}$, is inclined $22^{\circ}$ respect the horizontal, the tension is $250 \mathrm{kN}$, the diameter is $8 \mathrm{~cm}$, and the steel cross section is $7 \mathrm{~cm}^{2}$. The steel is in a petroleum wax, with polyethylene cover, and its mass is near $9.8 \mathrm{~kg} / \mathrm{m}$. The first normal frequency is $1.8 \mathrm{~Hz}$. A periodic force was applied, searching the resonance, to induce appreciable oscillations, without the SMA damper, and later with a NiTi SMA simple damper constituted by only a wire of $2.46 \mathrm{~mm}$ diameter and $414 \mathrm{~cm}$ length, from near the $20 \%$ of the cable to the concrete base. The damper was trained previously with 100 cycles at $0.01 \mathrm{~Hz}$ and full transformation, because this reduces the creep and extends the fatigue life. The NiTi wire was pre-stressed to 500 $\mathrm{N}$ before the application of the external force.

The frequency analysis shows the appearance of the $1.8 \mathrm{~Hz}$ resonance for the cable without the SMA, with an exponential decreasing amplitude when the applied force is stopped. When the experiment is repeated with the NiTi wire, the lowest modes appear at near $2.05 \mathrm{~Hz}$, but there is a splitting (there are at least two resonance frequencies near $2.05 \mathrm{~Hz}$, see fig. 2), and also more elevated frequencies appear in the Fourier analysis. Also, to obtain similar displacement amplitudes, the needed force is much higher for the cable with the NiTi wire, and the decreasing of amplitude with time when the applied force is stopped, is faster with the NiTi wire attached.

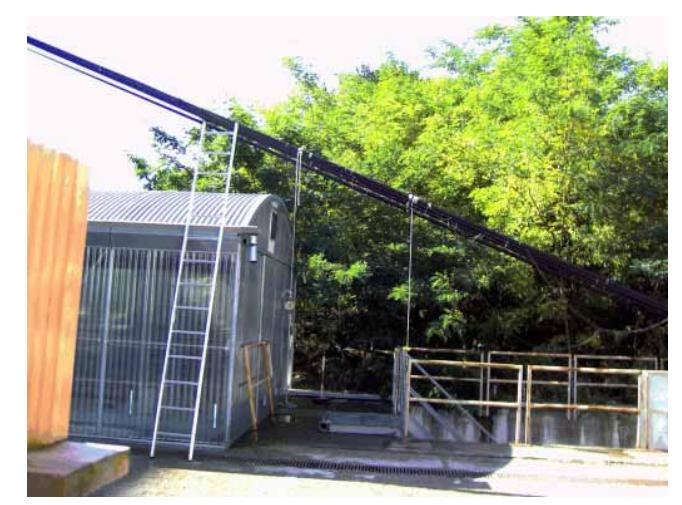

Fig. 1. Cables at ELSA (JRC, EU). At right, cable \#1.
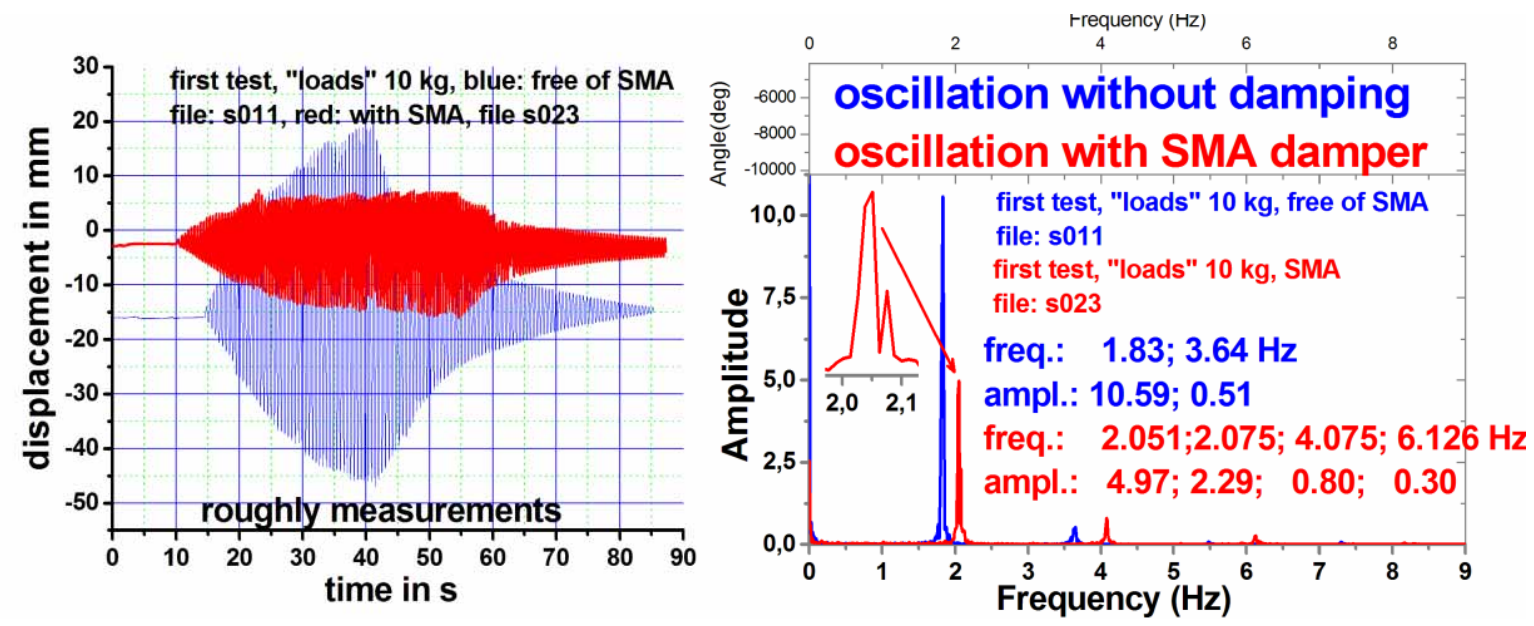

Fig. 2. Left: Forced vibrations of the ELSA cable \#1, exciting force at resonance $10 \mathrm{kp}(49 \mathrm{~N})$ of amplitude, without SMA damper (lower, blue) and with NiTi SMA damper (upper, red). Right: frequency analysis, sharp peaks without damper (blue), wider peaks with damper (red).

In the simulation with OPENSEES code, the cable was modelled as an "elasticbeamcolumn" of 100 elements, with Rayleigh damping of 0.01 (to take into account the wax and polyethylene effects). The effect of the number of elements on the computed normal modes was found to be rather small if more than 40 elements are 
considered; the effect of the Rayleigh damping was confirmed to be negligible, and the effect of the extreme boundary conditions (cable ends free to rotate or fixed in direction, as in the experimental cable the extremes are partially fixed against rotation) was found to be less than $4 \%$ on the frequency of the normal modes found by the linear approach with the OPENSEES code.

The NiTi SMA damper was simulated as the superposition of steel01 elastic-plastic elements in order to achieve a force-displacement behaviour similar to that measured in the wire. The excitation was an acceleration pulse of $15 \mathrm{~m} / \mathrm{s} 2$ up $(50 \mathrm{~ms})$, down $(100 \mathrm{~ms})$, and up $(50 \mathrm{~ms})$. The figure 3 shows the position of the point where the SMA wire would be subjected, without the SMA and with the SMA wire, as a function of time. The equilibrium position is slightly different, the initial oscillation amplitude is clearly reduced, and the time to decrease the amplitude is shortened with the SMA, in a rough agreement with the experimental results. The figure 4 shows the force-displacement of the experimental NiTi wire, and the simulation with elastic-plastic elements.
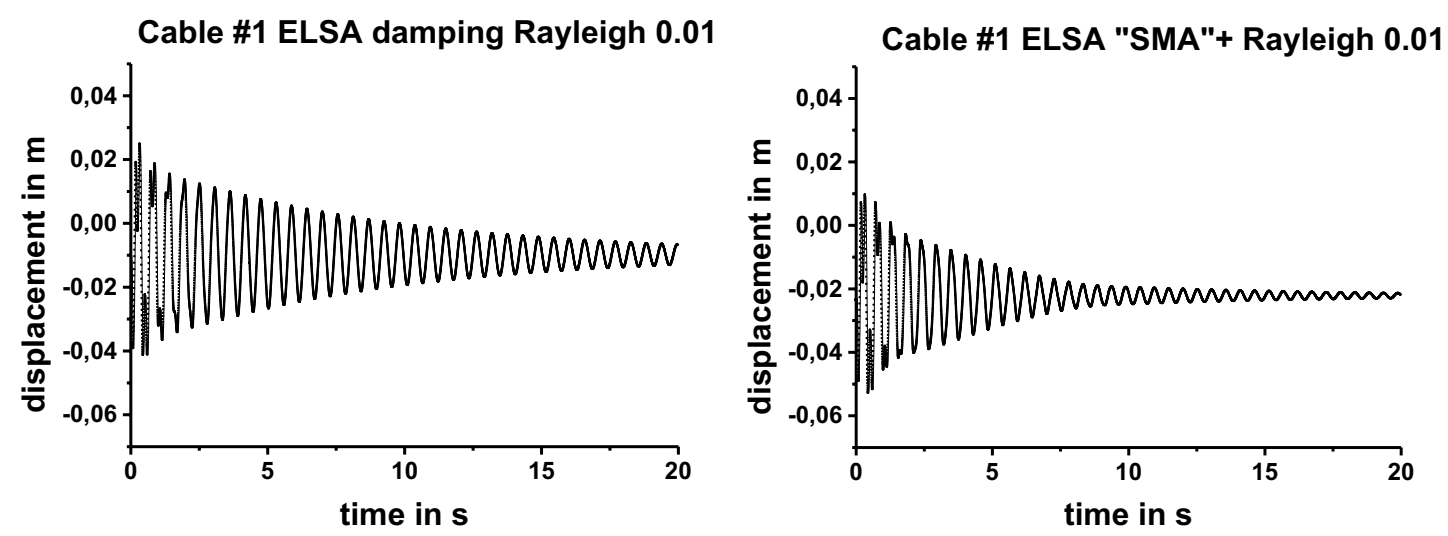

Fig. 3. Simulation results for cable \#1 at ELSA. Left: cable without SMA damper. Right: with SMA damper.
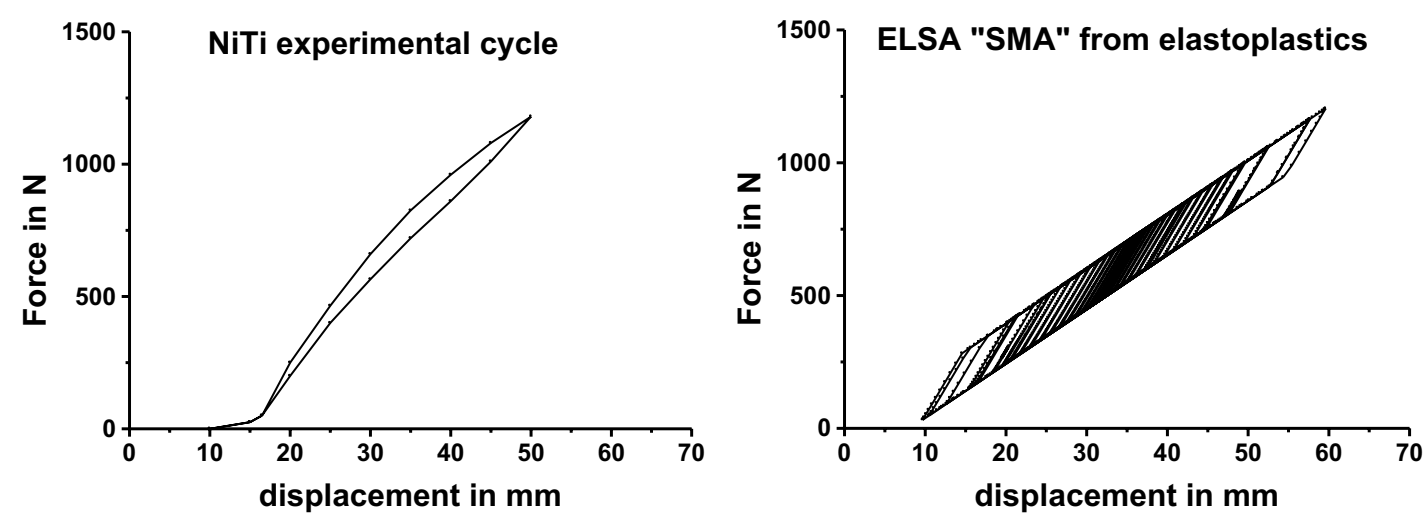

Fig. 4. Force-displacement diagrams for NiTi wire. Left: experimental. Right: model for simulation.

\subsection{Simulation of the Iroise bridge cable}

The Iroise bridge cable H3Q22, which links the platform of the bridge with a tower, with length $172 \mathrm{~m}$, diameter $18 \mathrm{~cm}$, and $79.6 \mathrm{~kg} / \mathrm{m}$, supporting a tension of $5.2 \mathrm{MN}$, was chosen for simulation. It is a steel cable with petroleum wax and a polyethylene cover. The ref. [9] reports on characteristics of the bridge and on measurements indicating a parametric wind induced oscillation with amplitude of the third normal mode near 10 $\mathrm{cm}$ in the antinodes, which represents vibration energy of some kJ. Due to the oscillations, some short hydraulic dampers were added in 2004, but some problems remain. As a first approach, we explored with simulations the possible effects of adding a SMA wire damper, from $20 \%$ of the cable to the platform of the bridge. The best option would be to place the dampers on the antinodes, but some compromise among performance, maintenance, and aesthetics limits the possibilities. A first simulation was done with ANSYS, with parameters for the SMA as stress to start the transformation, $\sigma_{\mathrm{ms}}=350 \mathrm{MPa}$, end of the transformation at $\sigma_{\mathrm{mf}}=500 \mathrm{MPa}$, end of the reverse transformation $\sigma_{\mathrm{af}}=150 \mathrm{MPa}$, strain to start the transformation $\varepsilon_{\mathrm{ms}}=0.01$, and strain to end the transformation $\varepsilon_{\mathrm{mf}}=0.05$. A length of $1 \mathrm{~m}$ was chosen for the simulated damper, because the expected displacement was less than $10 \mathrm{~cm}$ peak to peak, and the largest possible hysteresis from the material would be then profited. The 
remaining length from the cable to the platform was simulated as being of a thick steel cable. The system was excited with harmonic force, to obtain a $11 \mathrm{~cm}$ peak to peak amplitude at the anti-node, when no damper was in place. Then, with the same applied force, the effect of a $3.14 \times 10^{-7} \mathrm{~m}^{2}$ cross section SMA wire (thin wire) was computed, obtaining amplitude of $9.5 \mathrm{~cm}$ peak to peak at the anti-node. Further on, the effect of a $3.14 \times 10^{-6} \mathrm{~m}^{2}$ cross section SMA wire (2 mm diameter wire) was computed, obtaining amplitude of $8 \mathrm{~cm}$ peak to peak at the anti-node. In fact, in the later case, the deformation of the alloy is much lower than with the thin SMA wire, and very few energy is absorbed from the oscillation of the cable, the SMA wire tends to change the vibratory pattern by adding a "quasi-node". The optimisation of the damper position and its cross section is then a complex issue.

\section{Conclusions}

In this work we have analyzed the requirements for a SMA damper for stayed cables in bridges. The fatigue life conditions strongly the choice of SMA, and in fact, NiTi pseudo-elastic wire may accomplish the requirements for around 1000000 cycles if stresses are limited. Some experiments on the behaviour of an instrumented cable with a SMA damper were conduced in the cable \#1 at ELSA. The results show a change in normal modes, as well as a splitting in frequency, as corresponds to a strongly non-linear system. Also, a considerable decrease of oscillation amplitude is observed for constant exciting force amplitude, as well as a faster decrease of oscillation when the force is stopped.

The computer simulations, even using an approximate model for the SMA mechanically induced transformation, show characteristics coherent with the observations. However, to establish the optimal configuration of damper(s) needs further work in each case

\section{Acknowledgements}

Support from SMARTeR (ESF-MICINN BIA 2006-27041-E) project, and from C22/06 of 'Ministerio de Fomento' (Spain) is acknowledged. Previous supports for the long-time experiments came from CICYT (Spain).

\section{References}

[1] K. Otsuka, C.M. Wayman: Shape Memory Materials, Cambridge University Press, Cambridge, U.K., 1998. [2] J. Salichs, Z. Hou, M. Noori, "Vibration suppression of structures using passive shape memory alloy energy dissipation devices" J. of Intelligent Material Systems and Structures 12, p. 671-680 (2001).

[3] J.C. Wilson, M. Eeri, M.J. Wesolowsky, "Shape memory alloys for seismic response modification: a state of the art review", Earthquake Spectra 21 p. 569-601 (2005).

[4] V. Torra, A. Isalgue, F. Martorell, P. Terriault, F.C. Lovey: "Built in dampers for family homes via SMA: An ANSYS computation scheme based on mesoscopic and microscopic experimental analyses" Engineering Structures 29 p. 1889-1902 (2007).

[5] A. Isalgue, V. Torra, A. Yawny, F.C. Lovey: Metastable effects on martensitic transformation in SMA. Part VI. The Clausius-Clapeyron relationship. J. Thermal Anal. Calorim. 91 (2008) 3, 991-998

[6] V. Torra, A. Isalgue, F.C.. Lovey: "Microstructure and thermodynamics of the martensitic transformation". Canadian Metallurgical Quarterly, 39 p. 207-216 (2000).

[7] A. Isalgue, V. Torra, F.C. Lovey, J. Pelegrina: Low temperature aging behaviour of transformation temperatures in some Cu-based and NiTi SMA. ESOMAT 2009, Prague

[8] V. Torra, A. Isalgue, G. Carreras, F.C. Lovey, H. Soul, P. Terriault, B. Zapico: The SMA properties in civil engineering applications. The SMARTeR project: Use of SMA in damping of stayed cables for bridges. ESOMAT 2009, Prague.

[9] O. Boujard, S. Pernod, A. Berlioz, C.H. Lamarque: "Vibration of a cable stayed bridge: A peculiar resonance case", Proc. of the 2nd Inter. Conf. on Nonlinear Normal Modes and Localization in Vibrating Systems (Samos, Greece, 2006). 